\title{
Induced physical mutagenesis and its effect in cytological behavior of Ashwagandha (Withania somnifera (L.) Dunal)
}

\author{
T. Bharathi, S. Gnanamurthy, D. Dhanavel*, M. Ariraman \\ Division of Cytogenetics and Mutation Breeding, Department of Botany, Annamalai University, \\ Annamalai Nagar - 608 002, Tamil Nadu, India \\ *E-mail address: ddhana2005@yahoo.co.in
}

\begin{abstract}
The mitotic effect of physical mutagen gamma rays was observed in the root tip cells of Ashwagandha. The Chromosome analysis has been showed as an important tool for establish variability of the plant seed by use of physical mutagen gamma rays. The gamma rays have of low wavelength and high penetrable power. The plant has tremendous medicinal values and it is known from ancient times. The dry and well matured seeds of ashwagandha were irradiated with different doses of gamma rays viz., 5, 10, 15, 20, 25, 30, 35, 40, 45 and $50 \mathrm{KR}$ respectively. The chromosome number of control plant is $2 \mathrm{n}=48$. The gamma rays affect the normal cytological behavior of ashwagandha species. The chromosomal aberrations increase with increase in the doses of gamma rays to optimum level of $30 \mathrm{KR}$, because it causes changes in the chromosome structure, cellular structure and metabolism of plants. The chromosome aberration like, Sticky metaphase, Precocious moment chromosome, Fragments, Anaphasic bridge, Anaphasic laggard, Telophasic laggard. The present investigation was carried out to study the cytogenetic analysis of the species Withania somnifera. The chromosomal aberration increases with increase in the doses to optimum level (50 $\mathrm{KR}$ ) of physical mutagen gamma rays.
\end{abstract}

Keywords: Withania somnifera; chromosome; aberrations; gamma rays; KR (kilo rad)

\section{INTRODUCTION}

Withania somnifera belongs to the family Solanaceae, Ashwagantha is one of the important medicinal plants in India. There is an evidence of considerable morphological variation in the species and need for systematic work on the various morphological and geographical forms. The plants are generally erect branching shrubs, up to one meter in height. All the over ground parts, especially the stem, the vein, and the calyx, are covered with a sparsely hairy tomentum. The leaves are simple petiolate, ovate, exstipulate, entire acute and glabrous. The leaves on vegetative shoots are alternate and large while those on floral branches are opposite, flowers are short pedicellate 4-6 $\mathrm{mm}$ in diameter, gamosepalous, sepals 5- parted persistent with acute linear lobes. In the fruiting stage the calyx became enlarged, inflated and completely encloses the fruit. Corolla gamopetalous, 5- lobed, lobes spreading or recurved, acute pubescent and greenish yellow color stamens epipetalous, arising from the base of the petals; filaments slender; anthers innate and oval. Gynoecium 
syncarpous composed of minute swollen ovary subtended by a long slender style. Fruit, a berry enclosed in the green persistent calyx, is $5 \mathrm{~mm}$ in diameter, green when unripe, orangered when mature enclosing numerous small capsicum-like seeds (Kaul, 1957).

The plant has immense therapeutic uses in traditional and also in modern system of medicine. Ashwagantha roots are compared with ginseng roots for their restorative properties of vigour and vitality, hence the name "Indian ginseng". The whole plant, specially the leaves and root bark are abortifacient, adaptogen, antibiotic, aphrodisiac, deobstruent, diuretic, narcotic, strongly sedative and tonic (Bhandari, 1990) and (Chevallier, 1990). The fruit and seed are diuretic while latter are hypotonic. The roots of ashwagandha contain bioactive compounds with sedative and anti- inflammatory, anti-tumor, anti-bacterial and antispasmodic properties (Kumar et al., 2011) Ashwagandha acts mainly on the reproductive and nervous system having a rejuvenative effect on the body and is used to improve vitality and aid recovery after chronic illness. The medicinal properties of the species are due to its chemical constituents like alkoloides and withanolides (steroidal lactones) primarily in roots. Witheferin A is present in the leaf was reported by (Gupta et al., 1996) and (Das et al., 2009). Withaferin A and Withanolide A are the key components for therapeutic uses as they posses anticancerous, antioxidant and neuropharmacological properties apart from other uses.

The cytological analysis of both mitosis and meiosis is one of the most dependable indexes to estimate the potency of mutagen. Therefore, investigation on mitotic aberrations and their genetic consequences form an integral part of most of the mutation studies. It also provides a considerable clue to assess sensitivity of plants for different mutagen. Cytogenetic studies are necessary to obtain information regarding the role and effect of mutagen and in elucidating the response of genotypes to a particular mutagen. The cytomorphological variations induced by gamma rays in the root tip cells of Withania somnifera may be useful in studying the mode of action of the gamma rays as well as in inducing the cytomorphological mutation at later stage. The present investigation was carried out to assess the mutagenic effect of gamma rays on Withania somnifera (L.) Dunal by studying mitotic behavior of chromosomes in the root tip cells meristem of the seedlings.

Mutation is a sudden heritable change in an organism generally the structural change in genes. The term mutation was first introduced by (Hugo De Vries, 1901.,) in Oenothera lamarkiana. Mutation produced by changes in base sequences of genes (as a result of base pair transition or transversion, deletion, duplication or inversion etc.,) are known as gene mutation or point mutations. The agent that induce mutation are called mutagen. Gamma rays are one of the physical mutagen used in the field of mutation breeding. The technology of mutation breeding is a conventional method of plant breeding in order to supplement existing germplasm and to improve cultivars in specific traits. Improved varieties of many plants have been released through mutation breeding. Any mutagen that induces single base pair mutation or small deletion/ insertion is effective for tilling.

Gamma ray is one of the physical mutagen with low wavelength and high penetrating power and causes gene mutation in living organisms. Mutations are randomly distributed in the genome. A high degree of mutation saturation can be achieved with a mutagen like gamma rays that does not cause a lot of collateral DNA damage. 


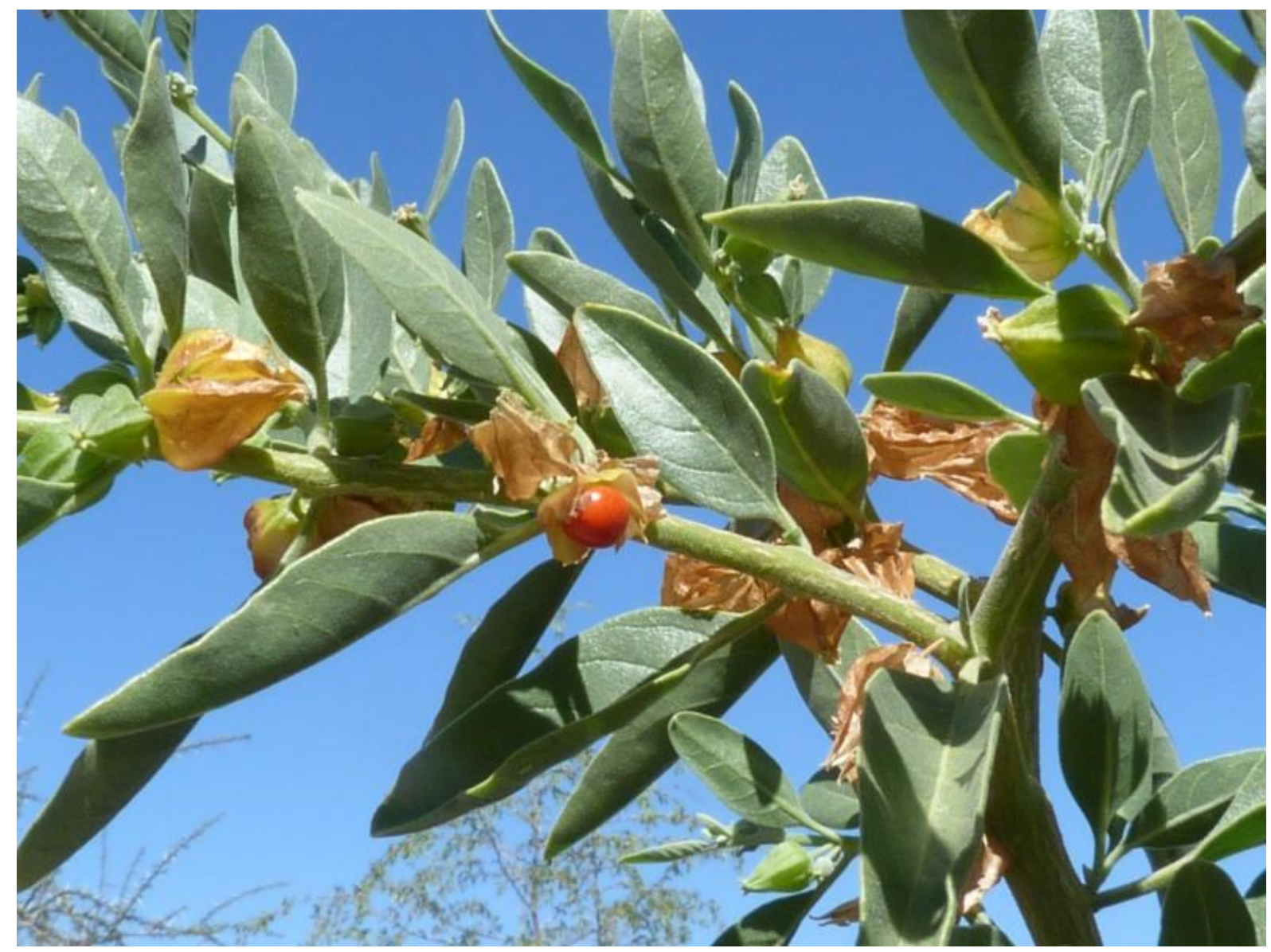

Photo 1. Withania somnifera (L.).

\section{MATERIALS AND METHODS}

The dry and dormant viable seeds of Ashwagandha variety Jawahar 20 were obtained from Tamil Nadu Agricultural University (TNAU) Coimbatore. Gamma rays are ionizing radiation having low wave length with high penetrable power; interact with atoms or molecules to produce free radicals in the cells. The radicals can damage or modify important components of plant cells. The source of gamma rays is Cobalt 60 , one of the labeled metals, which emit gamma rays.

The Ashwagandha seeds were treated with $(5,10,15,20,25,30,35,40,45$ and 50KR) of gamma rays. The untreated seeds were used as control. The seeds were germinated on moist filter paper in petridishes at $25 \pm 2{ }^{\circ} \mathrm{C}$ in BOD (Biological oxygen demand) incubator under dark conditions in cytogenetic and mutation breeding research laboratory, Department of Botany, Annamalai University and Annamalai Nagar till the emergence of radicals. The gamma rays irradiated seeds were also sown in pots for morphological studies. The root tips were collected in the morning time between 7.30 to 8.00 A.M, root tips of appropriate length $(0.5-1.0 \mathrm{~cm})$ were exised and after pretreatment, the root tips were washed 4-5 times in distilled water, carefully dried by absorbing the moisture and subsequently fixed in 1:3 glacial acetic acid- absolute ethanol mixture. The root tips were stored in $10 \%$ ethanol at 10 ${ }^{\circ} \mathrm{C}$ in a refrigerator for long term use, whenever needed. 
The root tips of Ashwagandha were taken from 1:3 Acetic-Alcohol and were thoroughly washed in distilled water for 2 or 3 times. The root tips were hydrolyzed in $1 \mathrm{~N}$ HCL for 8-10 min and the tips were kept at $60{ }^{\circ} \mathrm{C}$ in water bath, followed by washing with 3 changes of distilled water and then in iron alum for 5-10 min. Finally the root tips were stained in hematoxylin stain for 2-3 hours and then washed in distilled water. Then the root tips were transferred to $45 \%$ percentage acetic acid solution few seconds. The root tips were kept in a clear side and a cover slip was mounted over it, and later it was squashed. The cover slip was mounted by Canada balsam. Microphotographs were taken from freshly prepared slides using Trinocular Research Phase Contrast Microscope attached with Digital Olympus Camera.

\section{RESULTS}

The physical mutagen gamma rays cause physiological damage besides gene and chromosomal changes. Physiological damage mainly manifested as growth retardation and death is generally restricted to $M_{1}$ generation. The present study showed that gamma radiation did not have severe effect on percentage of seed germination in Ashwagandha. The germination is initiated by swelling of cells by hydration followed by enzymatic activation and metabolism. The irradiation treatment can cause damage to embryo inside the seeds. The seed germination is apparently unaffected by the embryo damage. However, embryo damage might become apparent only on the later stages of ontogenesis. This is evident from the results of the survival count where survival rate decreased with increasing levels of doses. When the doses of gamma rays increases the seedling height was reduced when compared to control.

Mitosis was perfectly normal in control plants. The maximum aberrations were found at higher dose of the mutagen. The chromosomal abnormalities were present in almost all the treatments. Mitotic abnormalities increased along with increasing dosage of gamma rays.

The effect of gamma rays has been studied on mitotic activities of the root meristems. Mitosis was normal in the control plants of Withania somnifera. The chromosome number of the Ashwagandha plant counted as $2 \mathrm{n}=48$. Lower dosage of mutagen revealed more or less normal pairing likes that of control. However, a consistent increase in the frequency of various types of chromosomal abnormalities was observed with increasing dose of gamma rays treatments.

\section{CHROMOSOMAL ABERRATIONS}

\section{1. Precocious movements of chromosome}

In the normal chromosome pairing there is no precocious moment of chromosomes occurs. If the homology of chromosome pairing is disturbed or spindle mechanism is disturbed or inactivated, one or few chromosome moves towards the pole from the equatorial metaphase stage. These type of precocious moment occur due to the effect of gamma rays. Precocious movement of chromosome was dominant at metaphase stage at $20 \mathrm{KR}$ and $25 \mathrm{KR}$ of gamma ray treatments. 


\section{2. Laggard Chromosome}

The failure of chromosomal moments as a result of spindle fiber discrepancies one or few of the chromosome lags behind the other chromosomes moving towards the poles and lead to the formation of laggard chromosome. The lagging chromosomes appeared in the anaphase and also in the telophase stage. In the anaphase and telophase stage laggards was observed at $25 \mathrm{KR}, 30 \mathrm{KR}$ and $50 \mathrm{KR}$ of gamma ray treatments.

\section{3. Chromosomal Bridge}

In the normal separation of chromosomes the chromosomes separated equally. Chromosomal bridges occur due to sister chromatid exchange followed by delayed or failure of their separation at later stages of anaphase and telophase chromosome. Thick and sticky bridge appeared due to stickiness of chromosomes. The dominant telophasic and anaphasic bridge was observed in $30 \mathrm{KR}$ and $45 \mathrm{KR}$ of gamma ray treatments.

\section{4. Stickiness of chromosomes}

Stickiness of chromosomes was caused due to the polymerization of nucleic acids caused by the irradiation of gamma rays. Sticky metaphase was present in higher dosages $15 \mathrm{KR}, 30 \mathrm{KR}$ and $35 \mathrm{KR}$ of gamma rays.

\section{5. Fragments}

Chromosomal fragment occurs due to the failure of broken chromosome to recombine. Fragment was observed in the metaphase stage of higher dosages $25 \mathrm{KR}$ of gamma ray treatments.

\section{DISCUSSION}

There is a gradual reduction in seed germination, seedling survival and seedling height. Similar results were obtained in Ashwagandha (Iqbal and Datta 2007). Generally, gradual reduction in germination percentage observed from lower to higher dosage of gamma ray treatments (Bharathi, 2013). The reduction in seedling height is due to the changes in hormonal levels such as auxins and ascorbic acid: physiological and biochemical disturbances (Gunckel, 1954, Singh, 1974); changes in enzyme activity and impaired mitosis in the meristamatic zone of growing seedlings. The similar results were suggested that it might also be due to a decrease in respiratory quotient in the irradiated seedlings (Blinks, 1952). In respect of gamma rays treatment, there was a proportionate reduction in germination of Andrographis paniculata with increased doses of gamma rays (Shroni, 2013)

The chromosome number of the Ashwagandha plant is 2n = 48 (Iqbal and Datta 2007 and Das, 2009). The reported chromosome number of Withania somnifera is 24, 48 and 72. (Mohan Ram and Kamini 1964,) had reported 2n $=24$ while $2 \mathrm{n}=48$ was reported by many authors (Mir et al., 2010, Kumar et al., 2011, Chatha 1998, Kaul 1957, Mohan Ram and Kamini 1964, Baquar 1965, Meige 1960). (Bir and Sidhu 1980) reported 2n $=72$ presumably a polyploidy number reported for this species. Chromosomal observation were reported by many workers, Sunflower (Elangovan, 1995), Black gram (Bandyopadhyay, 1983), Chilly (Dhamayanthi, 2000) Chick pea (Sharma 2004) and the physical mutagen gamma rays induced many mitotic abnormalities in Soybean (Pavadai, P 2013). The mutagen can cause 
physiological damage besides gene and chromosomal changes. Physiological damage, mainly manifested as growth retardation and death, is generally restricted to $\mathrm{M}_{1}$ generation. Such a phenomenon has been attributed to changes in hormonal levels such as auxins and ascorbic acid. Physiological and biochemical disturbances (Gunckel, 1954, Singh, 1974), change in enzyme activity and impaired mitosis in the meristematic zone of growing seedlings (Blinks, 1952). Similar results were suggested that it might also be due to a decrease in respiratory quotient in irradiated seedlings (Woodstock and Justics, 1967). In the view of these contradictory reports, the present investigation was conducted in Ashwagandha with sole objective to comprehend chromosome behavior by passing the gamma rays in the seeds. Mitotic aberrations were recorded by many authors in plants raised from seeds treated with different dosages of the gamma rays.

Precocious movements of chromosomes might have occurred due to disturbed homology for chromosome paring, disturbed spindle mechanism or in activation of spindle mechanism (Agarwal and Ansar 2001) in Vicia faba. Laggards were present in almost all treatments and occurrence of laggards in the present study has also been reported previously by many workers such as (Singh et al., 1999) in Vigna radiata, (Iqbal and Datta 2007), (Khan, et al., 2009.) The radiation can have direct effect on chromosomes. They may directly break chromosomes or alter one of the DNA bases or indirectly may initiate a chain of physical and chemical reactions (Dhanavel et al 2012) in Cowpea. Delayed terminalisation and or failure of chromosomal movements, following spindle fibre discrepancies have lead to lagging chromosomes. The fragments which appeared on the breakage of bridges, as result of spindle fibres functioning to pull the chromosomes towards poles, formed laggards (Kumar and Gupta et al., 2009) in Aswagantha.

Bridges occur due to sister chromatid chromosomes exchange followed by delayed or failure of their separation at later stages. Bridge formation observed by (Ahmad and Yasmin 1992), (Utsunomiya et al., 2002) and (Cequea et al., 2003). Bridges might have occurred as a result of delayed terminalization (Bipasha and Shella, 1992), unequal separation of chromosomes (Iqbal and Datta, 2007). The thick sticky bridges may be due to the stickiness of chromosomes. The stickiness interfered in the normal arrangement of chromosomes at metaphase and further led to their inability to separate, thus leading to sticky bridges. The spindle fibres pulled the chromosomes at metaphase and further led to their inability to separate, thus leading to sticky bridges. The spindle fibres pulled the chromosome towards the poles these bridges were broken into fragments, which either moved towards the poles or formed laggards and micronuclei (Kumar, 2003). Chromosomal bridges may also be due to the chromosomal stickiness and subsequent failure of anaphasic separation or may also be attributed to unequal translocation or in origin of chromosomal fragments. Lagging chromosome may be explained on the basis of abnormal spindle formation and failure of chromosome movement.

The fragments at metaphase may be due to the failure of broken chromosome to recombine. The Fragments might have arisen due to the stikiness of chromosome and the consequent failure of the arrival of chromatids at the poles. Fragments may also be acentric chromosomes formed as a result of inversion (Agarwal, 2001). Stickiness was one of the abnormalities found both in mitosis and in meiosis. It occurs due to disturbances of cytochemically balanced reactions by the effect of alkylating agents (Chidambaram et al., 2008). Bridges and laggards with or without fragments were found both at anaphase and telophase, bridges without fragments were found at higher dosages of mutagen. 


\section{PLATE-1}

\section{Chromosomal Aberrations in root tip cells of Ashwagantha}

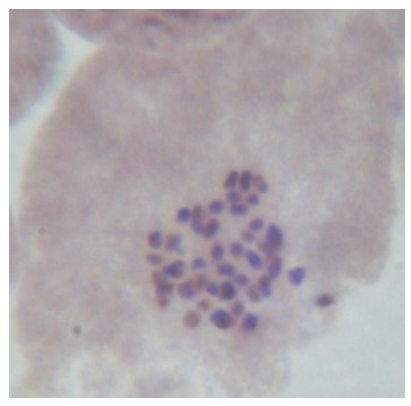

$\mathbf{A}$

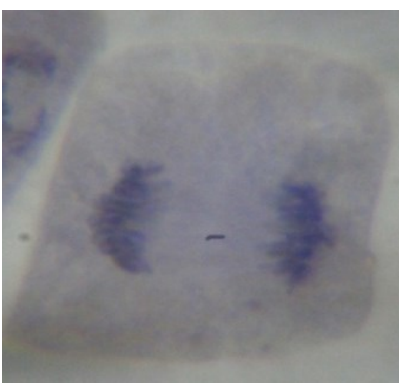

D

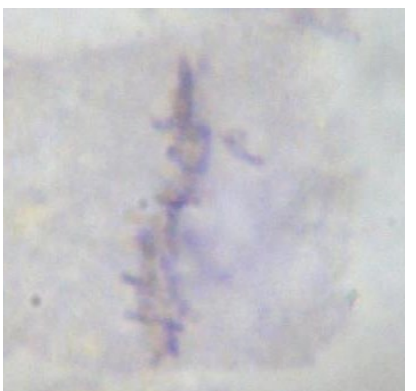

G

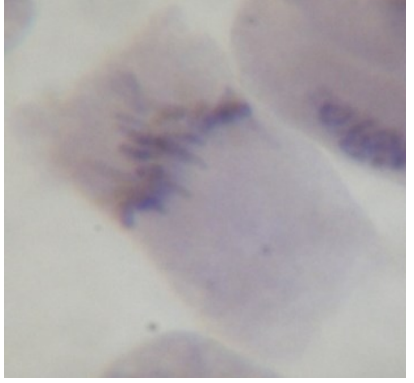

B

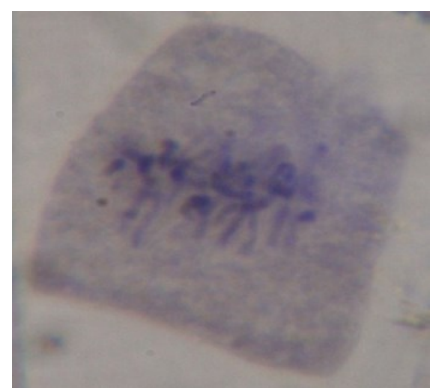

$\mathbf{E}$

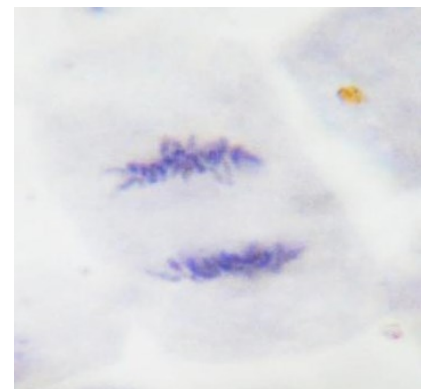

$\mathbf{H}$

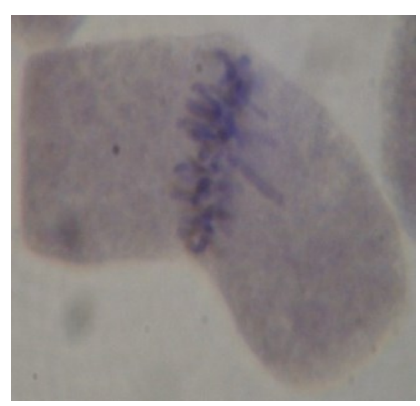

C

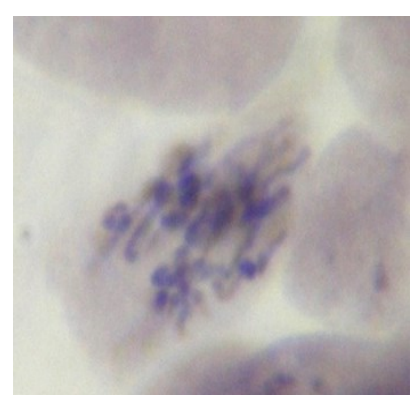

$\mathbf{F}$

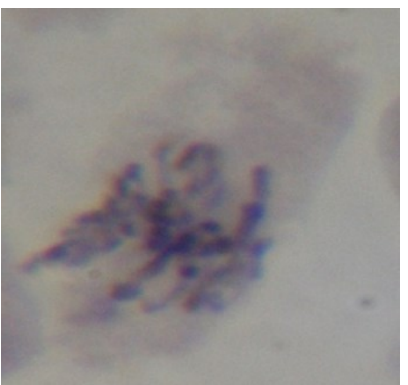

I

Figure A - Metaphase $2 \mathrm{n}=48$,

Figure B - Sticky metaphase

Figure $\mathrm{C}$ - Precocious moment chromosome,

Figure D - Telophasic laggard,

Figure E - Precocious moment,

Figure F - Sticky metaphase,

Figure G - Precocious moment,

Figure $\mathrm{H}$ - Anaphase chromosome,

Figure I - Sticky metaphase, 


\section{PLATE-2}

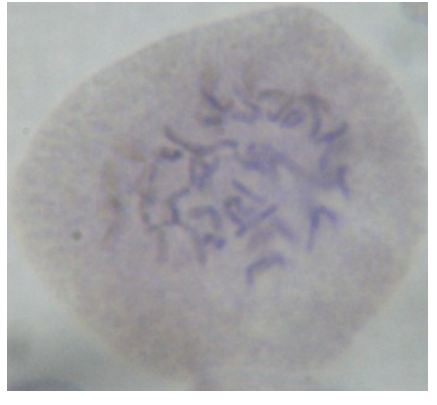

$\mathbf{J}$

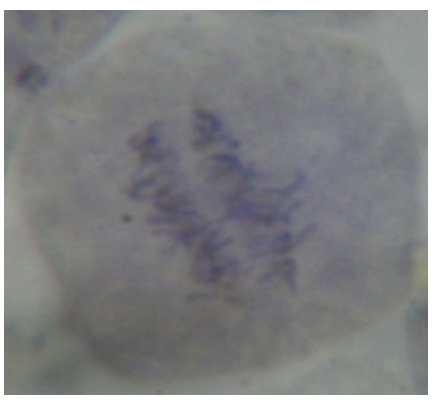

M

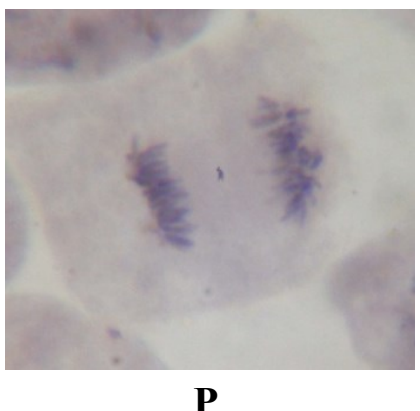

$\mathbf{P}$

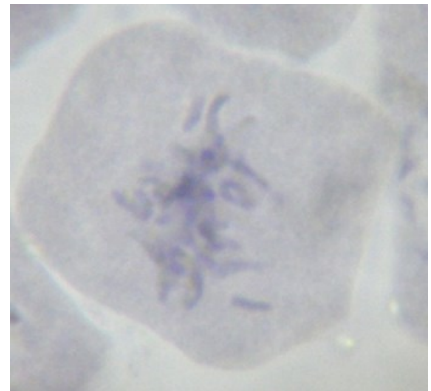

$\mathbf{K}$

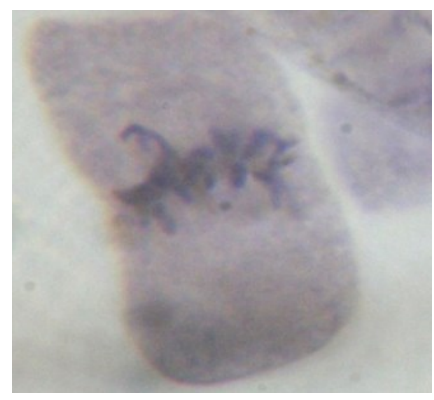

$\mathbf{N}$

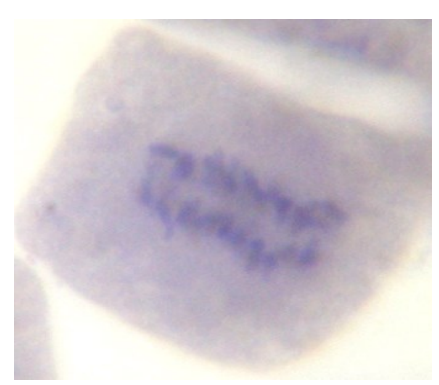

Q

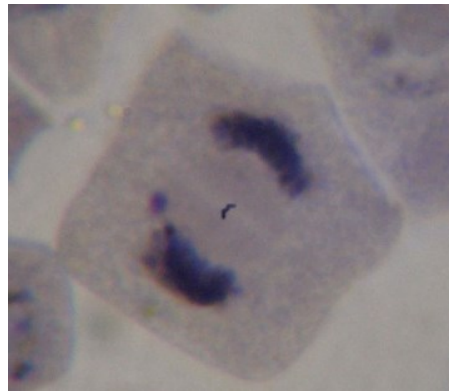

$\mathbf{L}$

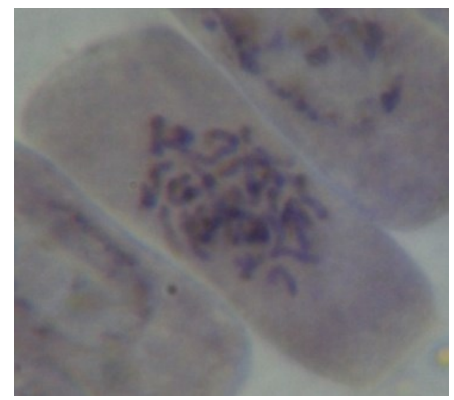

$\mathbf{O}$

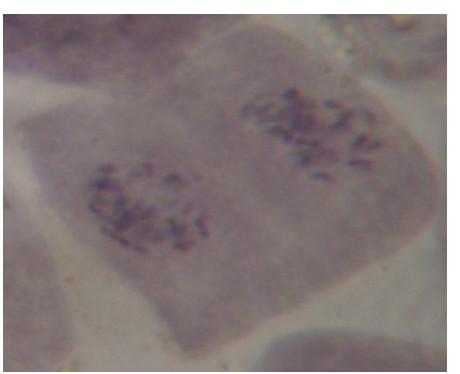

$\mathbf{R}$

Figure J - Control,

Figure K - Fragments,

Figure L - Telophasic laggard,

Figure $\mathrm{M}$ - Anaphasic bridge,

Figure N - Precocious moment,

Figure O - Control,

Figure $\mathrm{P}$ - Anaphasic laggard

Figure Q - Anaphasic bridge,

Figure R - Telophase chromosome. 


\section{CONCLUSION}

A dose dependent increase in the mitotic aberrations was observed in root tips of ashwagandha. It is clear from the observation that gamma rays caused more chromosomal damage in Ashwagandha and also a dose dependent reduction was observed in seed germination, seedling survival and plant height was also observed. Although gamma rays showed inhibitory effect on seed germination, plant height and seedling survival but the gamma rays have enough potential for induction of genetic variability and to exhibit quantitative and qualitative variations which can be favorably exploited by cytogenetic and plant breeders in improving the genotype of Withania somnifera (L.) and to improve the yield characters by cause of gene mutation.

\section{Acknowledgement}

The authors are thankful to the Head of the Department of Botany and authorities of Annamalai University for providing the necessary facilities and extend our heartfelt thanks to University Grants Commission, New Delhi for providing financial assistance under the BSR-SAP (Basic Science Research-Special Assistance Programme) for provided necessary facilities to carry out this work.

\section{References}

[1] Agarwal R., Ansari M. Y. K., J. Cytol. Genet. 2 (2001) 129-134.

[2] Ahmed S., Yasmin R., Cytologia 57 (1992) 155-160.

[3] Bandyopadhyay B., Bose S., 48 (1983) 13-19.

[4] Baquar S.R., S. Akhtar, A. Hussain, Botaniska Notiser 118 (1965) 289-298.

[5] Bhandari M.M. (1990). Flora of the Indian Desert, Scientific publishers, Jodhpur.

[6] Bharathi T., Gnanamurthy S., Dhanavel D., International Journal of Advanced Research 1(5) (2013) 136-141

[7] Bipasa C., Shella S., Mutat. res. 283 (1992) 287-294.

[8] Bir S., M. Sidhu (1980). Cytological Observations on Weed Flora of Patiala District, Punjab,” In: S.S.Bir, Eds., Recent Researches in plant science; Kalyani publishers, Ludhiana, 261-271.

[9] Blinks L. R., J. cellcomp. Physiol. 39 (1952) 11.

[10] Cequea H., Cequea D., Imery J., Nirichio M., Cytologia 68 (2003) 329-333.

[11] Chatha G. S., S. S. Bir, Plant sciences. 9 (1998) 199-256.

[12] Chevallier A. (1996). The Encyclopedia of Medicinal plants, Dorling Kindersley, London.

[13] Chidambaram A., Sundaramoorthy P., Murugan A., Sankar Ganesh K., Baskaran L., Iran J. Environ. Health Sci. Eng. 6 (1) (2008) 17-22.

[14] Das A., Datta A. K, Ghose S., J Trop Med Plants 10 (2009) 249-256. 
[15] Das A., Datta A. K., Ghose S., Bhattacharyya A., J. Trop. Med. Pl. 10 (2009) 225-230.

[16] Dhamayanthi K. P. M., Reddy V. R. K., Cytologia 65 (2000) 129-133.

[17] Dhanavel D., S. Gnanamurthy, M. Girija., Int. J. Curr. Sci. (2012) 245-250.

[18] Elangovan M., Selvaraj R., 25-26 ${ }^{\text {th }}$, Feb, 1995. In: proc. symp. On Frontiers in Biodiversity, Madras, India, Abstract No. 16.

[19] Gunkel J. E., Sparrow A. H., Brookhaven Symp. Biol. 6 (1954) 252-277.

[20] Gupta A. P., Verma R. K., Misra H. O., Gupta M. M., J. Medi. Aro. Plant Sci. 18 (1996) 788-790.

[21] Iqbal M., Datta A. K., J. Trop. Med. Pl. 8 (2007) 47-53.

[22] Iqbal M., Datta A. K., Cytologia 72 (2007) 43-47.

[23] Kaul K. N. (1957). The Orign, Distribution and Cultivation and cultivation of Ashwagantha the so called Withania somnifera of Indian Literature," Symposium on the Utilization of Indian Medicinal plants, Council of Scientific and Industrial Research, New Delhi, 7-8.

[24] Khan Z., Gupta H, Ansari M. Y. K., Choudry S., Biol and Med. 1(2) (2009) 66-69.

[25] Kothari S. K., Singh C. P., Vijay K. Y., Singh K., J. Hortic. Sci. Biotechnol. 78 (2003) 422-425.

[26] Kumar A., B. A., Mir D, Sehgal T. H. Dar, S. Koul, M. K. Kaul, S. N. Raina, G. N. Qazi., Plant systematics and Evolution 291(3-4) (2011) 141-151.

[27] Kumar G., Gupta P., Turk. J. Biol. 33 (2009) 123-128.

[28] Kumar G., Singh V., J. Ind. Bot. Soc. 82 (2003) 19-22.

[29] Miege J., Ann Fac Sci Univ Dakar 5 (1960) 75-85.

[30] Mir B. A., S. Loul A. Kumar M. K. Kaul, A. S. Soodan, S. N. Raina, Indian Journal of Biotecnolog 9 (2010) 325-328.

[31] Mohan Ram H. Y., I. Kamini, Phytomorphology 14 (1964) 574-587.

[32] Pavadai P., S. Gnanamurthy, D. Dhanavel, International Journal of Advanced Research 1(7) (2013) 223-228.

[33] Sharma V., Kumar G., Cytologia 69(3) (2004) 243-248.

[34] Shroni K., Bodele., Wall. Ex. Nees. 3(6) (2013) 55-57.

[35] Singh B. B., Rad Bot 14 (1974) 195-199.

[36] Singh G R., Sareen P. K., Sharan R. P., J. Cytol. Genet. 34 (1999) 21-23.

[37] Utsunomiya K. S., Bione N. C. P., Pagliarini M. S., Cytologia 67 (2002) 169-176.

[38] Woodstock and Justics, Rad. Bot. 7 (1967) 129-136. 